(2rossMark

Cite this: RSC Adv., 2017, 7, 10688

Received 9th January 2017

Accepted 2nd February 2017

DOI: $10.1039 / \mathrm{c} 7 \mathrm{ra00338b}$

rsc.li/rsc-advances

\title{
Soft colloidal lithography $\uparrow$
}

\author{
M. Weiler ${ }^{\mathrm{ab}}$ and C. Pacholski ${ }^{\star \mathrm{ac}}$
}

The exceptional properties of hydrogel colloids are exploited for the formation of tailor-made 2D colloidal crystals. The presented strategy facilitates the self-assembly of hydrogel colloids into highly ordered arrays in which the lattice constant and the diameter of the employed colloids can be independently adjusted by solely chemical means providing easy access to complex nanostructures.

Colloidal lithography is a well-established technique for fabricating nanostructured surfaces (reviewed e.g. in ${ }^{1-3}$ ). The appeal of this bottom-up approach is based on its simplicity, time- as well as cost-efficiency and its applicability to structuring large surface areas. In general it exploits the self-assembly of hard spheres such as silica ${ }^{4}$ or polystyrene ${ }^{5}$ nanoparticles into ordered two-dimensional arrays. Different methods were utilized for assembling and depositing the colloids onto surfaces such as the Langmuir-Blodgett approach ${ }^{6,7}$ pioneered by e.g. Möhwald, ${ }^{8}$ dip coating, ${ }^{9}$ spin coating, ${ }^{10,11}$ and lift-off from interfaces. ${ }^{12,13}$ Most often hexagonally close-packed colloidal monolayers were prepared but also other arrangements including loosely packed hexagonally ordered arrays ${ }^{14}$ and square arrays ${ }^{15,16}$ were realized by carefully choosing appropriate deposition parameters including ionic strength, $\mathrm{pH}$, and concentration of the colloidal solution. In order to generate loosely packed hexagonally ordered arrays of hard spheres very often previously deposited close-packed arrangements were reworked with reactive ion etching ${ }^{17}$ or thermal treatment. ${ }^{18}$ The latter provides more elaborated nanostructures. Also the degree of order of the resulting array depends strongly on the fabrication process and at least two requirements have to be fulfilled in order to obtain colloidal arrays composed of hard spheres with a minimal amount of defects: highly monodisperse spheres and a clean environment (if possible free of dust).

An elegant way to promote the formation of defect-free colloidal crystals is the utilization of soft spheres for their production. Their softness and compressibility facilitate the dissipation of defect energies over long distances. Thereby the

${ }^{a}$ Max Planck Institute for Intelligent Systems, Department of New Materials and Biosystems, Heisenbergstr. 3, 70569 Stuttgart, Germany. E-mail: cpachols@ uni-potsdam.de

${ }^{b}$ University of Heidelberg, Biophysical Chemistry, Im Neuenheimer Feld 253, 69120 Heidelberg, Germany

'University of Potsdam, Institute of Chemistry, Am Mühlenberg 3, OT Golm, 14476 Potsdam, Germany

$\dagger$ Electronic supplementary information (ESI) available. See DOI: $10.1039 / \mathrm{c} 7 \mathrm{ra00338b}$ formation of dislocations in the crystal lattices by point defects, resulting from e.g. contamination of the surface with dust particles, is prevented. ${ }^{19}$ For example, by assembling stimuliresponsive hydrogel microgels composed of poly $(\mathrm{N}$-isopropylacrylamide) (polyNIPAM) at an air/liquid interface and applying mechanical shear 2D colloidal crystals with exceptional long-range order on large areas were obtained. ${ }^{20}$ The assembly of hard spheres into hexagonally ordered, closepacked monolayers at air/liquid interfaces has already been extensively studied. ${ }^{21-24}$ Also the Langmuir-Blodgett technique was successfully transferred to arranging polyNIPAM nanospheres into highly ordered lattices. ${ }^{25}$ Moreover, this method allowed a very precise control of the polyNIPAM interparticle distance. However, the diameter of the hydrogel colloids in this publication was controlled by reactive ion etching even though it has already be shown for single polyNIPAM microspheres adsorbed on glass substrates that their diameter can be changed by immersion and drying in certain solvents. ${ }^{26}$

In this work, a new technique referred to as soft colloidal lithography (SCL) is presented which allows for preparing $2 \mathrm{D}$ colloidal crystals with independent control over lattice constants and colloid diameters by taking advantage of the remarkable properties of polyNIPAM colloids. Solely chemical stimuli supply the necessary external preconditions in order to vary both parameters in the resulting highly ordered, loosely packed colloidal array. Hence, SCL paves the road to the simple, fast, and cost-efficient fabrication of tailor-made 2D colloidal crystals which can be utilized to obtain highly desired photonic metamaterials.

The process of SCL is schematically shown in Fig. 1. Briefly, polyNIPAM microgels were wet-chemically synthesized according to Pelton et al. ${ }^{27}$ and adapted from Kim et al. ${ }^{28}$ (details of the synthesis can be found in the ESI†). Two different types of polyNIPAM microspheres were thereby obtained which were investigated throughout this work: on the one hand polyNIPAM colloids with a diameter of $\sim 1 \mu \mathrm{m}$ and on the other hand anionic polyNIPAM colloids with a diameter $\sim 2 \mu \mathrm{m}$. To guarantee the formation of highly ordered 2D colloidal crystals, polyNIPAM microspheres were pre-assembled at an air/liquid 


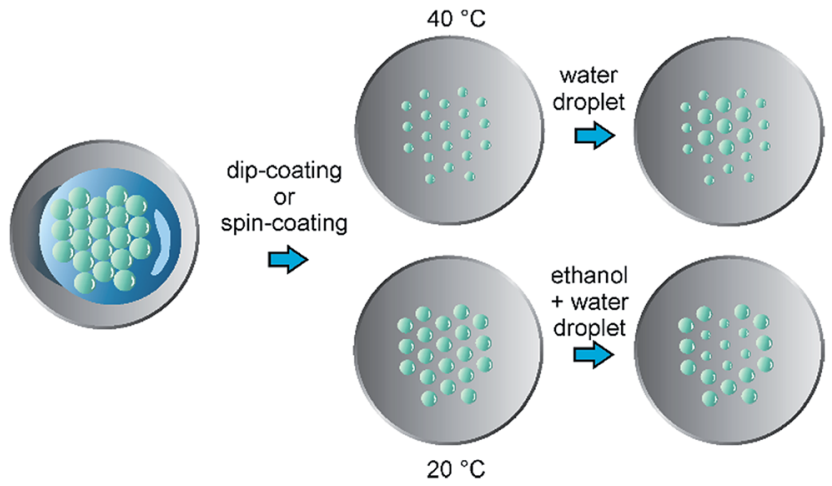

Fig. 1 Soft colloidal lithography (SCL). PolyNIPAM colloids are selfassembled into highly ordered arrays at an air/liquid interface and subsequently deposited onto glass substrates using dip coating or spin coating. The diameter of the polyNIPAM colloids was varied by the deposition parameters, e.g. the temperature, without changing the lattice constant of the 2D colloidal crystal. Localized tuning of the diameter of the polyNIPAM colloids was achieved by treating the substrate surface with liquids inducing swelling or collapse of the hydrogel.

interface by addition of ethanol to the colloidal dispersion. The resulting hexagonally ordered loosely packed array of polyNIPAM colloids was mechanically annealed and afterwards transferred onto cleaned glass cover slips using dip coating or spin coating. ${ }^{20}$

In Fig. 2 images of hexagonally arranged polyNIPAM arrays on glass substrates are displayed which were obtained by scanning electron microscopy (SEM) detecting backscattered electrons.

Both arrays were prepared using polyNIPAM colloids with a diameter of $\sim 2 \mu \mathrm{m}$ which were pre-assembled on a glass substrate and transferred onto a water surface at a defined temperature.

Then the polyNIPAM monolayer was deposited onto glass substrates via dip coating. During this process the temperature of the water on which the pre-assembled polyNIPAM colloids floated was changed from $20{ }^{\circ} \mathrm{C}$ (Fig. 2a) to $50{ }^{\circ} \mathrm{C}$ (Fig. 2b). Details of the procedure are given in the ESI (Fig. S2 $\dagger$ ).

The influence of only this one parameter on the resulting 2D colloidal crystal is striking. The resulting colloidal arrays are characterized by their high degree of order, low variation in
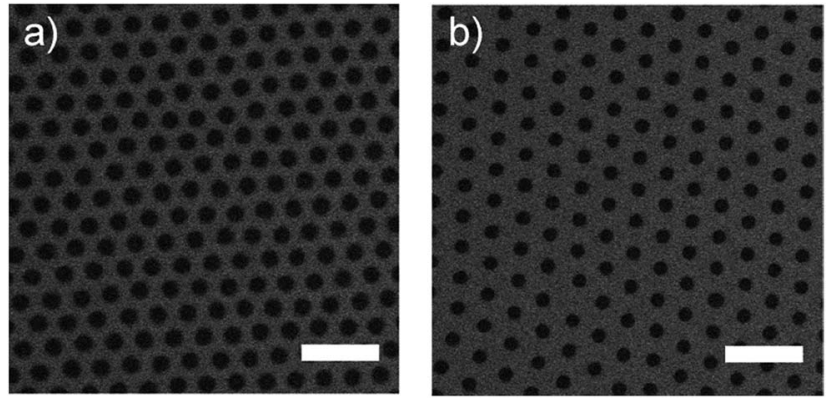

Fig. 2 Scanning electron micrographs of polyNIPAM colloids deposited onto glass substrates at different temperatures using dip coating: (a) deposition at $20^{\circ} \mathrm{C}$ and (b) deposition at $50{ }^{\circ} \mathrm{C}$. Scale bar: $5 \mu \mathrm{m}$. their periodicity, and their significant change in the diameter of the polyNIPAM colloids.

To get quantitative results for the geometrical arrangement of these arrays, the periodicity of the lattice and its degree of order was extracted from several SEM images according to a published method..$^{29,30}$ Briefly, the position of each polyNIPAM colloid in the array was determined using an algorithm implemented in the software ImageJ which served as basis for subsequent calculation of the radial distribution functions. All prepared colloidal arrays showed a very high degree of order (see Fig. S1a and $b_{\dagger} \dagger$ ). The lattice constant of the polyNIPAM colloids was determined to be $1.2 \mu \mathrm{m} \pm 0.1 \mu \mathrm{m}$ whereas the anionic polyNIPAM colloids assembled into an array with a higher periodicity of $2.1 \mu \mathrm{m} \pm 0.1 \mu \mathrm{m}$. The diameters of the polyNIPAM colloids on the surface were analyzed using the routine of ImageJ. If a deposition temperature of $20{ }^{\circ} \mathrm{C}$ was used, the diameter of the deposited colloid types was determined to be $0.72 \mu \mathrm{m} \pm 0.02 \mu \mathrm{m}$ and $1.15 \mu \mathrm{m} \pm 0.09 \mu \mathrm{m}$ for polyNIPAM and anionic polyNIPAM colloids, respectively. By changing the deposition temperature to $50^{\circ} \mathrm{C}$ a reduction in the diameter of both types of polyNIPAM colloids was achieved: $0.61 \mu \mathrm{m} \pm 0.04 \mu \mathrm{m}$ for polyNIPAM colloids and $0.83 \mu \mathrm{m} \pm 0.13$ $\mu \mathrm{m}$ for anionic polyNIPAM spheres. Similar results were obtained using spin coating which are presented in the ESI (Fig. S3 and $\mathrm{S} 4 \dagger)$. The large difference in the diameters of the polyNIPAM colloids at different temperatures is based on the stimuli-responsive properties of polyNIPAM which can react to small differences in its environment, including temperature, $\mathrm{pH}$, and ionic strength of the dispersion, with an abrupt volume change (reviewed e.g. in $^{31}$ ). This exceptional response of polyNIPAM is based on altering the balance between hydrophilic and hydrophobic interactions in the polymeric network. Also polyNIPAM colloids adsorbed to a glass surface change their volume (reviewed e.g. in $^{32}$ ). In Fig. S6 $\dagger$ AFM images of anionic polyNIPAM microspheres deposited at different temperatures onto glass surfaces are displayed. The cross-section demonstrates that not only the diameter of the anionic polyNIPAM colloids ('footprint') but also their height varies considerably: $82 \mathrm{~nm} \pm 3 \mathrm{~nm}$ for arrays fabricated at low temperatures and $160 \mathrm{~nm} \pm 7 \mathrm{~nm}$ for arrays prepared at high temperatures, for example. These results are in accordance with published values, which were obtained for similar experiments on the volume changes of polyNIPAM colloids on glass surfaces which were induced by other stimuli. ${ }^{33}$

However, the lattice constants of the obtained polyNIPAM arrays are not significantly influenced by the deposition temperature (Fig. S5 $\dagger$ ). This behaviour is intuitively unexpected but has already been reported for the assembly of large polyNIPAM colloids at oil/water interfaces. Here, polyNIPAM colloids change their shape and develop a flattened morphology based on their amphiphilic properties. Depending on the softness (cross-linking density) of the polyNIPAM colloids their peripheral shells can overlap to a different extent leading to a constant interparticle distance in the formed hexagonal array. ${ }^{34,35}$

Moreover, the diameters of polyNIPAM colloids on glass substrate surfaces were gradually changed. For this purpose 
a pre-assembled colloidal polyNIPAM monolayer was deposited onto the surface of a temperature-controlled water bath. Then the polyNIPAM monolayer was transferred onto a glass substrate using dip coating. During the dip coating process the temperature of the water bath was gradually changed. In Fig. 3 SEM images of highly ordered polyNIPAM arrays at different positions of the glass substrate, which correspond to increasing water bath temperatures, are displayed.

Variations in the colloidal diameter can be easily noticed. Quantitative results for the polyNIPAM microsphere diameters and the lattice constants are shown in Fig. 4a and b, respectively. Whereas the diameter of the deposited polyNIPAM colloids changes gradually, the variation in the interparticle distance is not significant. By trend the lattice constant seems to increase with increasing temperature. This observation could be attributed to a change in the density of the polyNIPAM microsphere array during dip coating. Zhang et al. and others already demonstrated that the density of a colloidal polyNIPAM monolayer determines the lattice constant of the $2 \mathrm{D}$ colloidal crystal. ${ }^{7,36}$

The diameter of polyNIPAM colloids absorbed on a glass surface can also be changed locally providing a chemical route

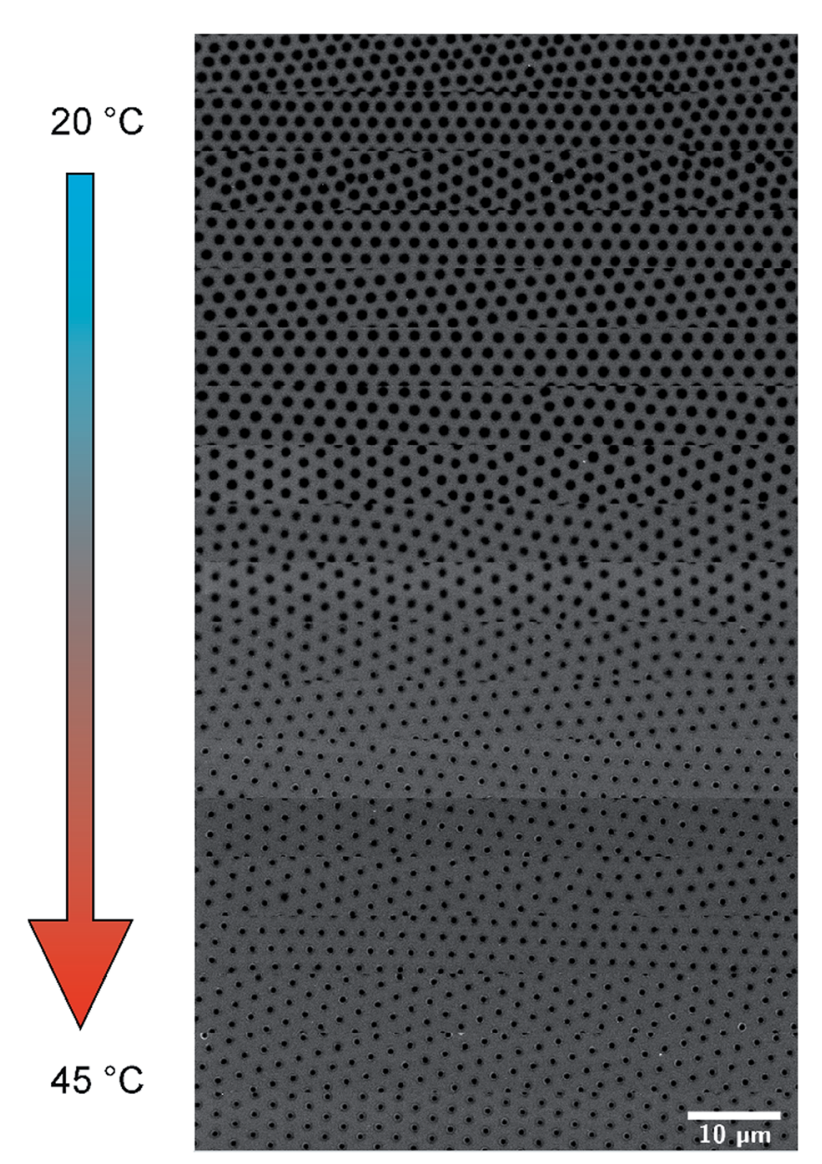

Fig. 3 SEM images of polyNIPAM colloids deposited onto glass using dip coating. During the deposition the temperature of the water, on which a pre-assembled polyNIPAM colloidal monolayer floated, was gradually changed. SEM images of the sample were taken at different positions of the glass substrate, reflecting different temperatures.
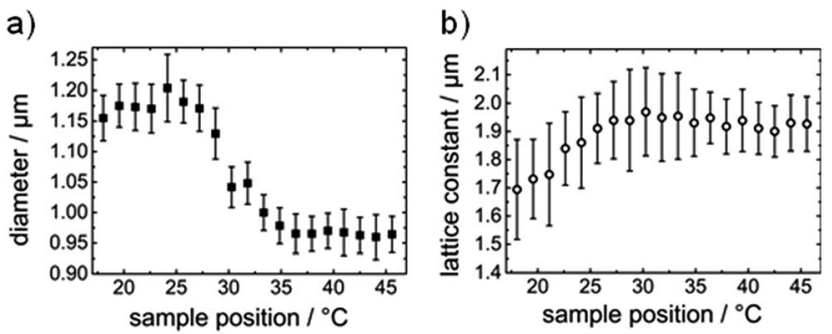

Fig. 4 Diameter of polyNIPAM colloids (a) and lattice constants (b) of prepared loosely packed colloidal arrays as a function of deposition temperature during dip coating.

to more complex structures. Fig. 5a shows a SEM image of a loosely packed polyNIPAM microsphere array which has been prepared at $20{ }^{\circ} \mathrm{C}$ and subsequently treated locally with a mixture of ethanol/bidest. Water containing $20 \mathrm{vol} \%$ ethanol, i.e. placing a droplet at RT onto the substrate surface followed by drying at RT. On the right hand side of the image the diameter of the polyNIPAM microspheres is significantly larger than on the left hand side. A histogram of the polyNIPAM diameters present on the substrate surface is displayed in Fig. $5 \mathrm{~b}$ and demonstrates the bimodal distribution. The process works also vice versa. PolyNIPAM colloids which have been deposited at $40{ }^{\circ} \mathrm{C}$ onto the substrate surface have a diameter of $\sim 0.8 \mu \mathrm{m}$ which can be changed to $\sim 1 \mu \mathrm{m}$ by water treatment at RT (Fig. 5c and d).

The lattice constant of the colloidal array is not influenced by locally applying water to the substrate surface and drying the sample at RT. Moreover, the swelling/collapse of polyNIPAM colloids deposited on glass substrates is reversible in accordance with published results on the thermoinduced behaviour of single polyNIPAM colloids adsorbed on surfaces. ${ }^{33}$ a)

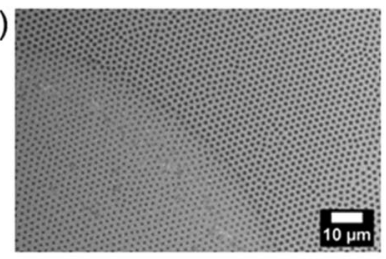

c)

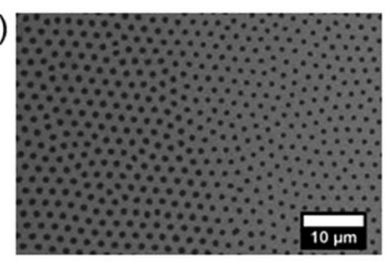

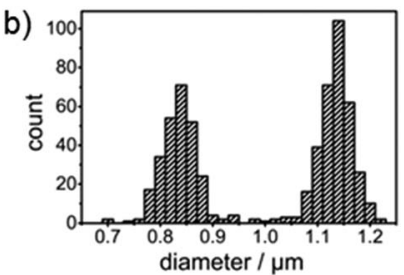

d)

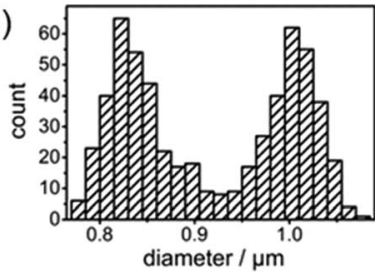

Fig. 5 SEM images and corresponding histograms of polyNIPAM colloids on glass substrates whose diameter was changed locally. (a) SEM image of polyNIPAM colloids deposited at RT and locally treated with 20 vol\% ethanol in water. (b) Distribution of the diameter of the polyNIPAM colloids shown in (a). (c) SEM image of polyNIPAM colloids deposited at $40{ }^{\circ} \mathrm{C}$ onto the glass substrates which have locally be swollen after applying a water droplet at RT. The corresponding histogram is shown in (d). 


\section{Conclusions}

To summarize, the first example of independent control of interparticle distance and particle diameter in highly ordered 2D colloidal crystals using solely chemical methods is demonstrated. Highly ordered hydrogel microsphere arrays were obtained by self-assembly at an air/liquid interface and the diameter of the colloids was controlled by the deposition protocol. By changing the temperature during the transfer of the hydrogel microgel monolayer onto the substrate surface the diameter of the colloids was successfully tuned gradually as well as over the whole substrate surface. Furthermore, by placing small droplets of a ethanol/water mixture onto a 2D colloidal crystal made of hydrogel microspheres, their diameter was locally changed suggesting a promising application of the presented method to the fabrication of photonic metamaterials using e.g. metal-assisted etching (MAE) of silicon.

\section{Acknowledgements}

M. Weiler and C. Pacholski acknowledge financial support from the German Federal Ministry of Education and Research (BMBF, project PhoNa, contract no. 03IS2101E) and the Max Planck Society.

\section{Notes and references}

1 P. Colson, C. Henrist and R. Cloots, J. Nanomater., 2013, 948510.

2 J. Y. Park, Korean J. Chem. Eng., 2014, 31, 541-547.

3 N. Vogel, C. K. Weiss and K. Landfester, Soft Matter, 2012, 8, 4044-4061.

4 W. Wang, B. H. Gu, L. Y. Liang and W. Hamilton, J. Phys. Chem. B, 2003, 107, 3400-3404.

5 S. M. Yang, S. G. Jang, D. G. Choi, S. Kim and H. K. Yu, Small, 2006, 2, 458-475.

6 K. U. Fulda and B. Tieke, Adv. Mater., 1994, 6, 288-290.

7 B. van Duffel, R. H. A. Ras, F. C. De Schryver and R. A. Schoonheydt, J. Mater. Chem., 2001, 11, 3333-3336.

8 R. M. Kenn, C. Bohm, A. M. Bibo, I. R. Peterson, H. Mohwald, J. Alsnielsen and K. Kjaer, J. Phys. Chem., 1991, 95, 2092-2097.

9 A. S. Dimitrov and K. Nagayama, Langmuir, 1996, 12, 13031311.

10 P. Jiang and M. J. McFarland, J. Am. Chem. Soc., 2004, 126, 13778-13786.

11 H. W. Deckman and J. H. Dunsmuir, J. Vac. Sci. Technol., B: Microelectron. Process. Phenom., 1983, 1, 1109-1112.

12 M. Kondo, K. Shinozaki, L. Bergstrom and N. Mizutani, Langmuir, 1995, 11, 394-397.
13 H. H. Wickman and J. N. Korley, Nature, 1998, 393, 445-447. 14 L. Isa, K. Kumar, M. Muller, J. Grolig, M. Textor and E. Reimhult, ACS Nano, 2010, 4, 5665-5670.

15 C. D. Dushkin, G. S. Lazarov, S. N. Kotsev, H. Yoshimura and

K. Nagayama, Colloid Polym. Sci., 1999, 277, 914-930.

16 H. Cong and W. X. Cao, Langmuir, 2003, 19, 8177-8181.

17 C. Haginoya, M. Ishibashi and K. Koike, Appl. Phys. Lett., 1997, 71, 2934-2936.

18 Z. C. Zhou, Q. F. Yan, Q. Li and X. S. Zhao, Langmuir, 2007, 23, 1473-1477.

19 A. S. Iyer and L. A. Lyon, Angew. Chem., Int. Ed., 2009, 48, 4562-4566.

20 S. B. Quint and C. Pacholski, Soft Matter, 2011, 7, 3735-3738. 21 C. Geng, L. Zheng, J. Yu, Q. Yan, T. Wei, X. Wang and D. Shen, J. Mater. Chem., 2012, 22, 22678-22685.

22 M. Kondo, K. Shinozaki, L. Bergstroem and N. Mizutani, Langmuir, 1995, 11, 394-397.

23 J. Yu, C. Geng, L. Zheng, Z. H. Ma, T. Y. Tan, X. Q. Wang, Q. F. Yan and D. Z. Shen, Langmuir, 2012, 28, 12681-12689.

24 J. Yu, Q. F. Yan and D. Z. Shen, ACS Appl. Mater. Interfaces, 2010, 2, 1922-1926.

25 M. Rey, R. Elnathan, R. Ditcovski, K. Geisel, M. Zanini, M. A. Fernandez-Rodriguez, V. V. Naik, A. Frutiger, W. Richtering, T. Ellenbogen, N. H. Voelcker and L. Isa, Nano Lett., 2016, 16, 157-163.

26 M. Richter, M. Hunnenmorder and R. V. Klitzing, Colloid Polym. Sci., 2014, 292, 2439-2452.

27 R. H. Pelton and P. Chibante, Colloids Surf., 1986, 20, 247256.

28 J. S. Kim, N. Singh and L. A. Lyon, Angew. Chem., Int. Ed., 2006, 45, 1446-1449.

29 R. Rengarajan, D. Mittleman, C. Rich and V. Colvin, Phys. Rev. E: Stat., Nonlinear, Soft Matter Phys., 2005, 71, 016615.

30 F. H. Kaatz, A. Bultheel and T. Egami, J. Mater. Sci., 2009, 44, 40-46.

31 H. Kawaguchi, Polym. Int., 2014, 63, 925-932.

32 S. Wellert, M. Richter, T. Hellweg, R. von Klitzing and Y. Hertle, Z. Phys. Chem., 2015, 229, 1225-1250.

33 A. Burmistrova, M. Richter, C. Uzum and R. von Klitzing, Colloid Polym. Sci., 2011, 289, 613-624.

34 M. Destribats, M. Eyharts, V. Lapeyre, E. Sellier, I. Varga, V. Ravaine and V. Schmitt, Langmuir, 2014, 30, 1768-1777.

35 M. Destribats, V. Lapeyre, M. Wolfs, E. Sellier, F. LealCalderon, V. Ravaine and V. Schmitt, Soft Matter, 2011, 7, 7689-7698.

36 G. Zhang, D. Wang, Z.-Z. Gu, J. Hartmann and H. Möhwald, Chem. Mater., 2005, 17, 5268-5274. 